\title{
Classificador baseado em Aprendizado Profundo para identificação de estratégias de alocação de espectro em Redes Ópticas Elásticas
}

\author{
Guilherme Silva $^{1,2}$, Lucas Costa $^{1}$, André Drummond ${ }^{1}$ \\ ${ }^{1}$ Departamento de Computação - Universidade de Brasília (UnB) \\ Brasília - Brasil \\ ${ }^{2}$ Departamento de Engenharia Elétrica - Universidade Estadual do Piauí (UESPI) \\ Piauí - Brasil
}

\begin{abstract}
Elastic Optical Networks provide better spectrum allocation flexibility and scalability than traditional Wavelength Division Multiplexing networks and are suitable to support the increasing demand for Internet traffic. Currently, these networks use routing and spectrum allocation algorithms with static strategies. The natural tendency of evolution is the use of adaptive strategies, according to network behavior. The first step is to create a classifier that can recognize whether the adopted heuristics are being effective at any given time, the focus of this work. The deep learning classifier obtained an accuracy of $98 \%$. The results obtained open the way for a new class of algorithms for Elastic Optical Networks, in which decision making can be done adaptively.
\end{abstract}

Resumo. As redes ópticas elásticas proporcionam uma flexibilidade e escalabilidade na alocação de espectro superior às tradicionais redes baseadas em multiplexação por divisão de comprimento de onda, sendo adequadas para suportar a crescente demanda do tráfego da internet. Atualmente, os algoritmos de roteamento e alocação de espectro dessas redes utilizam estratégias fixas. A tendência natural de evolução desses algoritmos é a utilização de estratégias adaptativas, de acordo com o comportamento da rede. Para isso, alguns passos são necessários. O primeiro deles é a criação de um classificador capaz de identificar as estratégias de alocação de espectro que estão sendo utilizadas, foco desse trabalho. $O$ classificador baseado em aprendizado profundo obteve acurácia de $98 \%$. Os resultados obtidos abrem caminho para uma nova classe de algoritmos para redes ópticas elásticas, nos quais a tomada de decisão pode ser feita adaptativamente.

\section{Introdução}

As redes de telecomunicações são fonte de uma enorme quantidade de dados heterogêneos. Essas informações podem ser recuperadas através dos rastros das redes, alarmes de falhas em equipamentos, indicadores de qualidade de sinal (como a taxa de erro de bit Bit Error Rate - BER), dados comportamentais de usuários etc. Para extrair informações importantes e tomar decisões a respeito do melhor funcionamento da rede a partir desses dados faz-se uso de algumas ferramentas matemáticas. Dentre elas tem-se destacado o aprendizado de máquina (Machine Learning - ML), o qual torna possível analisar os 
dados da rede e, a partir daí, realizar configurações automaticamente e de maneira assertiva. No contexto das redes ópticas a adoção de técnicas de ML tem sido motivada pelo enorme crescimento de sua complexidade, uma consequência da grande quantidade de parâmetros utilizados atualmente, tais como: configurações de roteamento, formato de modulação, taxa de símbolo, esquemas de codificação adaptativa, espaçamento flexível de canais, dentre outros [Musumeci et al. 2018, Mata et al. 2018].

ML é um ramo da Inteligência Artificial que parte da ideia de que, a partir do acesso a dados relevantes, máquinas podem aprender como resolver um problema específico [Goodfellow et al. 2016]. Essa abordagem existe há pelo menos 3 décadas, porém apenas recentemente a infraestrutura necessária para sua correta adoção tem se tornado disponível, ou seja, uma vasta quantidade de dados e um poder de processamento capaz de lidar com eles. Nesse contexto, um subgrupo do ML tem ganho destaque: o aprendizado profundo (Deep Learning - DL). Com a utilização de técnicas de DL adequadas é possível inferir, a partir de dados coletados nas redes, características importantes que não são facilmente detectadas.

Com base nessas características o DL começou a ser usado no planejamento da infraestrutura óptica que irá suportar a crescente demanda de tráfego de internet. Atualmente, grandes esforços têm sido aplicados para desenvolver novas tecnologias que viabilizam maior capacidade de transmissão nas grandes redes de transporte e, nesse contexto, as Redes Ópticas Elásticas (Elastic Optical Networks - EONs) têm ganho destaque. As EONs fazem uso do provisionamento de enlaces ópticos de maneira dinâmica para utilizar os recursos da rede de forma eficiente e flexível [Costa and Drummond 2017].

As redes ópticas elásticas enfrentam ainda alguns desafios para sua implantação, tanto em termos de hardware quanto em termos de software. Nesse último, por exemplo, a utilização de estratégias estáticas no processo de roteamento e alocação de espectro (Routing and Spectrum Allocation - RSA) não condiz com o cenário dinâmico de uma rede óptica[Lacerda et al. 2017]. A dinamicidade no estabelecimento e encerramento de circuitos ópticos, bem como a imprevisibilidade de tráfego futuro, pode desestabilizar o estado da rede óptica e prejudicar a eficiência dos algoritmos RSA [Chen et al. 2018].

Mesmo com a discussão a respeito das EONs, o termo exaustão (capacity crunch) tem se tornado cada vez mais frequente quando discute-se a respeito dos rumos da Internet. Ela ocorre quando os recursos para prover a velocidade desejada, a confiabialidade e outros requisitos da rede se tornam insuficientes com o aumento da demanda. Segundo [Waldman 2018], isso não significa uma catástrofe para a Internet, mas sim o "novo normal". A analogia que o autor utiliza são as ruas engarrafadas nos horários de pico de grandes cidades, quando as pessoas simplesmente se adaptam a conviver com isso.

Dessa forma, em algum momento a carga de tráfego na rede vai ser tão alta que a Internet vai chegar a exaustão. Nesse contexto, as métricas de taxa de bloqueio de circuito e taxa de bloqueio de banda assumem um papel importante para medir a probabilidade de exaustão da rede e consequentemente a eficiência de um algoritmo de engenharia de tráfego. Ou seja, considerando que a rede vai chegar em algum momento à exaustão, um algoritmo eficiente é aquele que retarda o máximo possível que isso ocorra, e uma das formas de mensurar isto são as taxas de bloqueio. Vários algoritmos RSA foram propostos na literatura e suas taxas de bloqueio são conhecidas. Como comentado anteriormente, 
esses algoritmos utilizam estratégias fixas em um cenário dinâmico. Dessa forma, a pergunta que permeia este trabalho é: seria possível, através de técnicas de Deep Learning, escolher adaptativamente às estratégias de roteamento e alocação de espectro durante o funcionamento da rede melhorando a utilização de seus recursos instalados e contribuindo para a diminuição de sua probabilidade de exaustão?

A complexidade dessa hipótese nos remete a alguns outros pontos que precisam ser esclarecidos previamente. Nesse contexto, quatro perguntas guiam esta pesquisa:

- É possível identificar algoritmos RSA utilizados em uma rede EON a partir de amostras do estado de alocação do espectro?

- É possível criar um classificador genérico capaz de identificar novos algoritmos?

- Essa classificação provê informações sobre a eficiência, em termos de taxa de bloqueio, do algoritmo?

- Com base nessas informações de eficiência das estratégias de roteamento e alocação de espectro adotadas, é possível criar um algoritmo eficiente de RSA que atue adaptativamente?

O presente trabalho representa o início do desenvolvimento dessa pesquisa e busca responder a primeira pergunta elencada acima, que já um desafio em termos matemáticos e computacionais. Utilizando técnicas de DL, o classificador proposto consegue identificar 8 estratégias diferentes de alocação de espectro com acurácia de 98\%. Assim, acreditase que esse trabalho é o passo inicial para o desenvolvimento de uma nova classe de algoritmos em EON: algoritmos que utilizam estratégias adaptativas durante sua execução com foco na redução da taxa de bloqueio.

\section{Conceitos Básicos}

As Seções a seguir apresentam uma breve contextualização teórica sobre EON e os conceitos básicos de DL como modo de fornecer uma melhor embasamento sobre a pesquisa.

\subsection{Redes Ópticas Elásticas}

Redes ópticas baseadas em multiplexação por divisão de comprimento de onda (Wavelength Division Multiplexing - WDM) utilizam para transmissão grades de frequência de tamanho fixo com granularidade de $50 G h z$ por comprimento de onda. Para cada demanda de tráfego uma grade inteira precisa ser alocada, mesmo que ela seja inferior à capacidade de acomodação de um comprimento de onda. Com o objetivo de melhorar o aproveitamento dos recursos de uma rede óptica, o paradigma EON surgiu com proposta de tornar a rede mais flexível, adotando uma arquitetura composta de grades de frequência que podem acomodar de maneira adaptável as demandas, sem causar desperdício de espectro. Similar aos algoritmos de roteamento e atribuição de comprimento de onda (Routing and Wavelength Assignment - RWA) de redes WDM, as EONs utilizam algoritmos de roteamento e alocação de espectro (RSAs). A restrição de continuidade de comprimento de onda, característica de algoritmos RWA, é substituída pela restrição de continuidade de espectro nos algoritmos RSA. Adicionalmente, outra restrição assume um papel importante: a contiguidade de espectro, que indica a necessidade que as grades de frequência (slots) sejam alocadas de forma adjacentes para a criação de circuito óptico.

As EONs permitem acomodar diferentes formatos de modulação de acordo com a distância de transmissão, e, nesse caso, uma nova restrição é adicionada ao algoritmo, 
sendo denominado de RMLSA (Routing, Modulation Level, and Spectrum Allocation). Mais recentemente, redes EON têm sido investigadas em cenários de fibras com múltiplos núcleos (Multi-Core Fiber - MCF), sendo chamadas de EONs com multiplexação por divisão espacial (Spatial Division Multiplexing - Elastic Optical Network - SDM-EON). Neste cenário o problema RMLSA passa ser chamado de RMSCA (Routing, Modulation, Spectrum and Core Allocation) [Tode and Hirota 2016].

Para este trabalho vamos considerar a existência de apenas um núcleo na fibra. Nesse contexto, os algoritmos RMLSA utilizaram uma abordagem sequencial, ou seja, irão resolver inicialmente o problema do roteamento, em seguida resolver o problema da modulação e, por fim, resolver o problema da alocação de espectro. Nessa primeira etapa da pesquisa vamos fixar a modulação como sendo a BPSK (Binary Phase-Shift Keying) e o roteamento como sendo o menor caminho (algoritmo de Dijkstra). Dessa forma, os algoritmos investigados irão divergir apenas com relação à alocação de espectro.

Atualmente os algoritmos RSA utilizam estratégias pré-definidas tanto para roteamento quanto para alocação. Essas estratégias são estáticas ou variam de acordo com alguma métrica. Para o roteamento, por exemplo, muitos algoritmos calculam as rotas mais curtas e escolhem uma delas. De maneira similar, quanto à alocação de espectro, muitos algoritmos utilizam a estratégia First-Fit para todas as demandas. Outros algoritmos usam, por exemplo, estratégias que variam de acordo com a demanda: First-Fit para demandas de baixa largura de banda e Last-Fit para demandas com alta largura de banda.

Usualmente a literatura divide a elaboração de um algoritmo de engenharia de tráfego EON em duas categorias: de forma sequencial; ou de forma integrada [Chatterjee et al. 2015]. Algoritmos que tratam o problema RMLSA ou RMSCA ${ }^{1}$ de forma sequencial buscam dividir o problema em partes, conforme apresentado na Figura 1. Inicialmente têm-se a escolha da rota (problema " $R$ "). Normalmente algoritmos sequenciais escolhem a rota com base na menor distância ou para as k-menores distâncias entre a origem e o destino da requisição de tráfego. No entanto, podem também escolher rotas baseado em alguma métrica, como por exemplo a rota que tem mais slots livres, ou seja, menos fragmentada. Em seguida, quando uma SDM-EON é considerada, têm-se o problema da escolha dos núcleos das fibras que irão compor a rota $R$, (problema " $C$ "). Em geral, escolhe-se o núcleo por meio de alguma política de escolha, tal como First-Fit ou Random-Fit. Posteriormente, para ambos os casos, é necessário escolher o nível de modulação que o algoritmo irá adotar no circuito óptico (problema " $M L$ " ou " $M$ "). Normalmente, utiliza-se a modulação mais eficiente que atenda os parâmetros de qualidade de transmissão (Quality of Transmission - QoT) pré-estabelecidos. Os modelos mais utilizados são: o alcance máximo transparente; e a utilização de modelos que mensuram as imperfeições e interferências da camada física na potência do sinal óptico. Por fim, têmse como última etapa a escolha da frequência de espectro na fibra, ou seja, a sequência de slots contíguos que irão compor o circuito óptico (problema " $S$ ").

Para os algoritmos que tratam o problema de forma integrada, as soluções procuram resolver os problemas em uma única etapa, ou mesmo agregando etapas. Por exemplo, realizando o roteamento, seleção de formato de modulação e a alocação de espectro simultaneamente. Resolver os problemas RMLSA ou RMSCA de forma sequencial é

\footnotetext{
${ }^{1}$ Somente quando SDM-EON é considerado.
} 


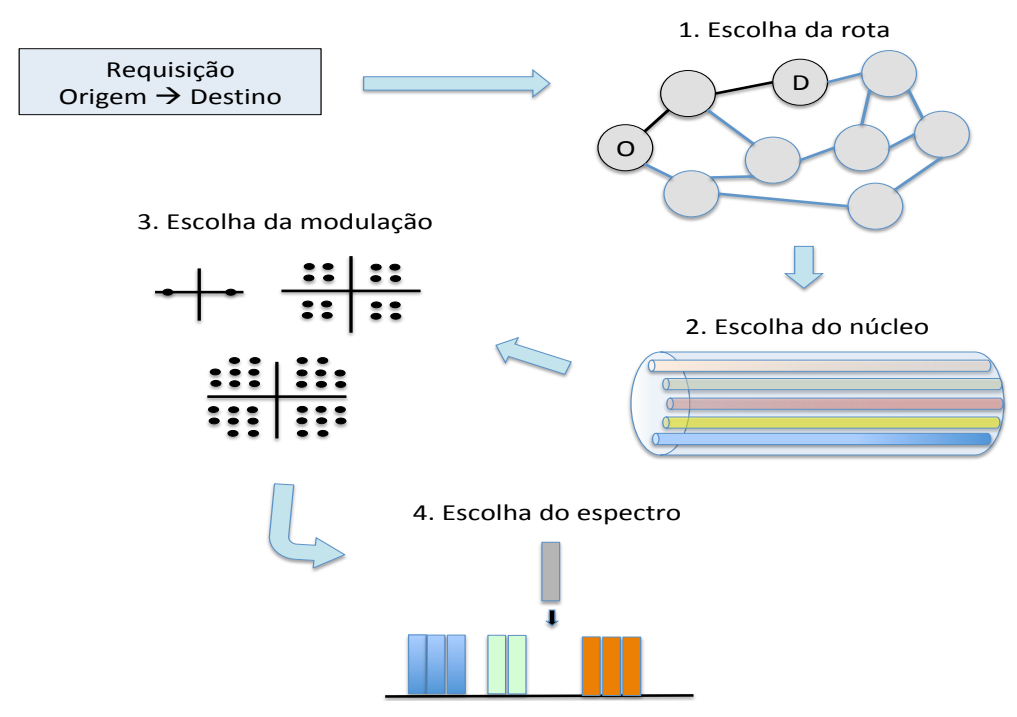

Figura 1. Forma sequencial de atuação de algoritmos EON

computacionalmente mais simples do que resolver de forma integrada.

As estratégias de alocação de espectro investigadas nesse trabalho são: First-Fit (FF), Last-Fit (LF), Random-Fit (RF) [Chatterjee et al. 2015], ExactFit (EF) [Rosa et al. 2012], Best-Fit (BF) [Leiva et al. 2014], Pseudo Partitioning (PP) [Wang and Mukherjee 2012], Dedicated Partition (DP) [Tessinari et al. 2018] e FirstLastExactFit (FLEF) [Chatterjee et al. 2016]. Para o cenário descrito na Seção 4.1, a probabilidade de bloqueio e o bloqueio de banda desses algoritmos considerando a solução de roteamento de menor caminho estão mostrados nas Figuras 2(a) e 2(b), respectivamente. Essas taxas, conforme explicado na Seção 1, serão importantes para a identificação da qualidade das estratégias utilizadas. Por exemplo: i) sabe-se que a estratégia RF apresenta resultados ruins enquanto a estratégia FLEF apresenta bons resultados; e ii) sabe-se também da semelhança no comportamento em termos de taxa de bloqueio das técnicas FF e LF. Esse tipo de informação será utilizado nas próximas etapas da pesquisa para aperfeiçoamento do classificador proposto.

As estrategias de alocação de espectro podem ou não serem baseadas na demanda. A descrição do comportamento de cada estratégia de alocação de espectro avaliadas neste trabalho é apresentado a seguir:

\section{Estratégias de alocação não baseadas na demanda:}

First-Fit (FF): Estratégia mais utilizada na literatura, em que escolhe-se a faixa de slots disponíveis com os menores índices.

Last-Fit (LF): Equivalente ao First-Fit. Escolhe-se a faixa de slots disponíveis com os maiores índices.

Random-Fit (RF): Escolhe-se a faixa de slots disponíveis de forma aleatória.

Exact-Fit (EF): Procura escolher uma faixa de slots livres com a quantidade de slots igual à solicitada, caso não encontre, busca escolher a faixa com a maior diferença entre os slots livres e solicitados.

Best-Fit (BF): Escolhe-se a faixa de slots disponíveis cuja a quantidade de slots é a mais próxima da quantidade de slots solicitada. 


\section{Estrategias de alocação baseadas na demanda:}

Pseudo Partitioning (PP): Procura utilizar First-Fit para determinadas classes de serviço e Last-Fit para outras.

Dedicated Partition (DP): Busca segregar as demandas em determinadas faixas do espectro, podem utilizar o compartilhamento dessas faixas ou não. Ex.: Demandas que requisitam poucos slots ficam com os menores índices.

FirstLastExactFit (FLEF): Cria grupo de pares com rotas disjuntas e não disjuntas para decidir qual estrategia de alocação de espetro vai ser utilizado. Para rotas disjuntas utiliza-se a estrategia FirstExactFit ${ }^{2}$, enquanto para rotas não disjuntas utiliza-se a estrategia LastExactFit ${ }^{3}$.

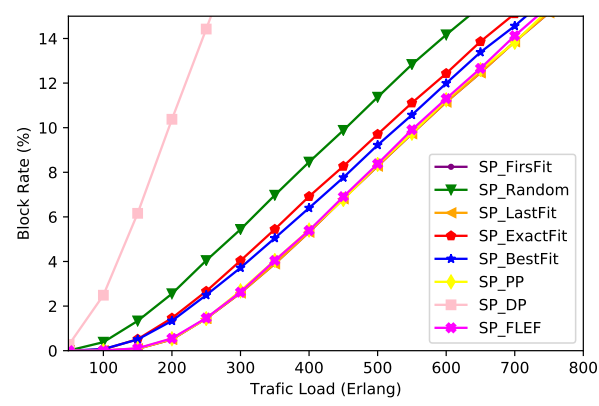

(a) Taxa de Bloqueio.

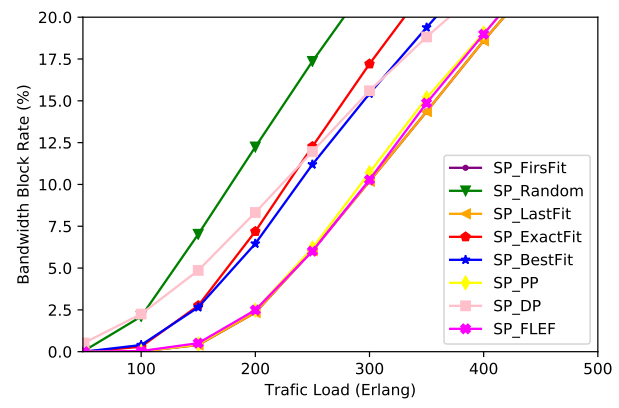

(b) Taxa de Bloqueio de Banda.

Figura 2. Resultados dos algoritmos de alocação de espectro avaliados.

\subsection{Deep Learning}

Denomina-se de Aprendizado de Máquina a utilização de algoritmos para extrair informações de dados brutos e representá-los através de algum tipo de modelo matemático. Pode-se usar este modelo para fazer inferências a partir de outros conjuntos de dados. No contexto desse trabalho, algoritmos supervisionados são o foco: tem-se variáveis de entrada $(x)$ e uma variável de saída $(Y)$, e utiliza-se um algoritmo para aprender uma função que mapeie a variável de saída a partir dos dados de entrada. O aprendizado supervisionado é útil para resolver problemas de classificação, ou seja, problemas nos quais a variável de saída é uma categoria, e não um número real. Dentre os algoritmos inclusos nesse grupo pode-se citar a Máquina de Vetores de Suporte (Support Vector Machine - SVM) e a Floresta Aleatória (Random Forest). Porém um tipo em especial vem se destacando: as redes neurais artificiais (RNs). Apesar de terem sido propostas na década de 1950, as redes neurais vem ganhando grande destaque recentemente devido à grande quantidade de dados disponíveis e ao aumento no poder de processamento das máquinas. Assim, com o aumento na complexidade das RNs, surgiu uma nova subclasse de técnicas denominadas de aprendizado profundo (DL). Como será explicado na Seção 4, as entradas desta pesquisa têm dezenas de milhares de atributos, o que remete à utilização de técnicas de DL.

\footnotetext{
${ }^{2}$ Procura escolher uma faixa de slots livres com a quantidade de slots igual à solicitada, caso não encontre escolhe-se a faixa de slots disponíveis com os menores índices.

${ }^{3} \mathrm{O}$ mesmo que o FirstExactFit só que caso não encontre escolhe-se a faixa de slots disponíveis com os maiores índices.
} 


\section{Trabalhos Relacionados}

Nos últimos anos alguns trabalhos utilizando aprendizado de máquina em EON têm sido propostos na literatura, principalmente no que diz respeito a estimativa de QoT, estabelecimento de conexão, gestão de falhas e design de topologias virtuais, como será descrito a seguir.

Estimar a qualidade de transmissão do sinal antes do estabelecimento do circuito é uma técnica interessante no projeto de redes ópticas. Modelos analíticos costumam estimar grosseiramente a QoT, fazendo com que as redes tenham que introduzir grandes margens no orçamento de potência dos circuitos ópticos, subutilizando os recursos da rede. Nesse sentido, os autores [Barletta et al. 2017] propõem um modelo baseado em ML para determinar se determinada rota, modulação e espectro (RMLSA) teria uma taxa de erro de bits em níveis aceitáveis. Os atributos utilizados na predição foram o volume de tráfego, o formato de modulação, o comprimento total do circuito óptico, o comprimento do enlace mais longo e o número de enlaces. Já em [Yan et al. 2017] foi demonstrada uma rede óptica baseada em SDN que utiliza mecanismos de ML para prever a relação sinal-ruído óptica (Optical Signal-To-Noise Ratio - OSNR) dos enlaces. Nessa mesma linha, [Proietti et al. 2018] tornou o cenário ainda mais complexo adicionando múltiplos domínios e propôs uma arquitetura EON baseada em redes neurais para estimar a QoT dos circuitos ópticos por domínio.

No âmbito do estabelecimento de conexão, em [Araujo et al. 2015] foram utilizadas redes neurais artificiais para prever a probabilidade de bloqueio das redes ópticas. Utiliza-se como parâmetros as propriedades topológicas da rede, informações da camada física, carga e algoritmo utilizado. Embora o estudo tenha sido feito para WRONs (Wavelength-Routed Optical Networks), os autores sugerem que o modelo pode ser adaptado para EONs. Já em [Jia et al. 2016] foi implementado uma rede neural para prever o tempo de chegada e o tempo de espera de futuras conexões em EONs. Com base nas informações dos prováveis enlaces que serão estabelecidos, foi proposto um algoritmo dinâmico de roteamento e alocação de espectro com taxas de bloqueio satisfatórias. Ainda no contexto de estabelecimento de conexão, [Chen et al. 2018] propõe um framework para roteamento, modulação e alocação de espectro em EONs baseado em Aprendizado Profundo por Reforço (Deep Reinforcement Learning - DRL). Essa técnica de DL tem se mostrado promissora no que diz respeito ao roteamento de tráfego, segundo [Boutaba et al. 2018].

Considerando o aspecto da gestão de falhas, a perda de pacotes devido a problemas na camada física de redes ópticas é um problema de suma importância para os operadores de rede, tendo em vista que impacta diretamente nos acordos de nível de serviço (Service Level Agreement - SLA) e acarreta em prejuizos financeiros. Nesse contexto, [Vela et al. 2017] propõe dois algoritmos baseados em aprendizado de máquina: BANDO e LUCIDA. O primeiro, localizado nos nós ópticos, detecta mudanças de BER nos links e comunica ao segundo algoritmo, localizado em um controlador central. Com essa arquitetura foi possível prever a violação máxima de BER dias antes da interrupção da conexão, permitindo o planejamento eficiente na reconfiguração da rede.

Do ponto de vista do design de topologias virtuais, em [Morales et al. 2017] os autores utilizam redes neurais na predição de tráfego para realizar a reconfiguração da topologia virtual da rede. O mesmo grupo de pesquisa, em [Velasco et al. 2017], estendeu 
os modelos matemáticos e algoritmos utilizados no trabalho anterior para o cenário EON, focando na resolução de problemas de otimização relacionados ao design, operação e reotimização das mesmas.

Dessa forma, de acordo com a pesquisa realizada, os trabalhos que aplicam DL em EONs ainda estão incipientes, deixando uma vasta margem a ser explorada. Nesse contexto, este trabalho é o ponto de partida para uma área praticamente inexplorada: a construção de uma solução RSA que utiliza um classificador baseado em deep learning para ajustar dinamicamente a estratégia de alocação de espectro em algoritmos para EONs com o objetivo de reduzir a taxa de bloqueio.

\section{Classificador}

Nesta seção descreveremos o ambiente de simulação, o pré-processamento realizado, a entrada da RN e seus hiperparâmetros.

\subsection{Ambiente de Simulação}

As simulações foram realizadas pelo simulador ONS [Costa and Drummond 2019]. Cada simulação foi realizada cinco vezes apresentando intervalos de confiança com $95 \%$ de confiabilidade.

Em cada simulação foram geradas $10^{5}$ chamadas com diferentes níveis de granularidade: 12,5 Gbps, 25 Gbps, 50 Gbps, 100 Gbps, 200 Gbps e 400 Gbps. O processo de chegada das chamadas segue a distribuição de Poisson com origem e destino distribuídos uniformemente para todos os pares de comunicação da rede.

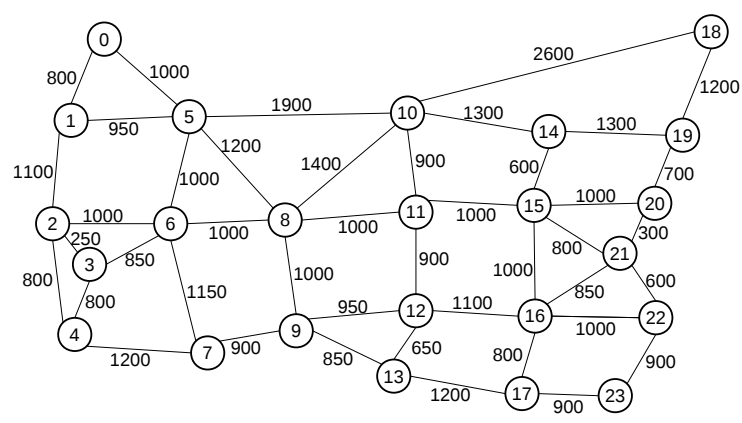

Figura 3. Topologia USANet

A topologia considerada nas simulações foi a USANet com 24 nós e 86 enlaces, mostrada na Figura 3. Cada enlace tem uma banda de $4 \mathrm{THz}$, dividida em faixas de frequência de 12,5 GHz, o que resulta em 320 slots por fibra [Walkowiak et al. 2018, Muhammad et al. 2015]. Considera-se 2 slots de banda de guarda entre circuitos ópticos adjacentes para evitar interferência entre eles. Cada nó na topologia é equipado com transmissores e receptores suficientes para acomodar todas as solicitações de tráfego. A modulação utilizada foi a BPSK.

\subsection{Entrada da Rede Neural e pré-processamento}

O primeiro passo foi definir o estado da rede. De acordo com o número de enlaces ópticos e slots mencionados na Seção anterior, pode-se representar a rede como uma matriz de dimensão $(86,320)$, conforme pode-se observar a representação esquemática na Tabela 1. 
O simulador ONS foi adaptado para que sua saída contenha essa matriz binária, e o valor de cada célula identifique a ocupação do slot. Mais especificamente:

- Uma célula com valor "1"determina que aquele slot está alocado para algum circuito óptico (incluindo banda de guarda);

- E uma célula com valor "0"indica que aquele slot está vago.

\section{Tabela 1. Tabela representando a organização da rede óptica como uma matriz de 86 links e 320 slots.}

\begin{tabular}{|c|c|c|c|c|c|c|c|c|c|c|c|c|c|c|c|c|}
\hline & \multicolumn{15}{|c|}{ Slot } \\
\hline & & 1 & 2 & 3 & 4 & 5 & 6 & 7 & 8 & 9 & $\ldots$ & 316 & 317 & 318 & 319 & 320 \\
\hline \multirow{6}{*}{$\begin{array}{l}\stackrel{8}{\tilde{\Xi}} \\
\frac{\pi}{\tilde{I}}\end{array}$} & 1 & 0 & 0 & 0 & 1 & 1 & 1 & 0 & 0 & 0 & $\ldots$ & 1 & 0 & 0 & 1 & 1 \\
\hline & 2 & 1 & 1 & 1 & 1 & 1 & 0 & 0 & 1 & 1 & $\ldots$ & 0 & 1 & 1 & 0 & 0 \\
\hline & 3 & 1 & 1 & 0 & 0 & 1 & 1 & 1 & 0 & 0 & $\ldots$ & 0 & 0 & 0 & 0 & 0 \\
\hline & & $\ldots$ & $\ldots$ & $\ldots$ & $\ldots$ & $\ldots$ & $\ldots$ & $\ldots$ & $\ldots$ & $\ldots$ & $\ldots$ & $\ldots$ & $\ldots$ & $\ldots$ & $\ldots$ & $\ldots$ \\
\hline & 85 & 1 & 1 & 0 & 0 & 1 & 1 & 1 & 0 & 0 & $\ldots$ & 0 & 0 & 1 & 1 & 0 \\
\hline & 86 & 1 & 1 & 1 & 0 & 0 & 0 & 1 & 1 & 0 & $\ldots$ & 1 & 0 & 0 & 1 & 1 \\
\hline
\end{tabular}

Redimensionando a matriz bi-dimensional para apenas uma dimensão obtém-se um vetor com 27.520 posições, que representa o que pode-se chamar de estado da rede. Pode-se imaginar o estado da rede como uma foto de como a rede está organizada em determinado instante. Ele é a entrada da rede neural proposta.

Cada requisição que chega na rede óptica, se ela for atendida, muda o estado da rede. Denomina-se de momento $n$, a chegada da enésima requisição. Durante a operação da rede guarda-se o estado em vários momentos. Este trabalho considera 100 estados da rede, uniformemente distribuídos entre a requisição 1.000 (momento 1.000) e o momento 100.000 .

\subsection{Classes}

Em um modelo de aprendizado de máquina as classes são as possíveis saídas do classificador. Pretende-se observar um estado de rede (entrada da RN) e classificá-la como algum dos algoritmos nos quais a rede neural foi treinada.

Como mencionado na Seção 2.1, o foco inicial é em algoritmos RMLSA dos quais são variados apenas as estratégias de alocação de espectro. A estratégia de roteamento e escolha de modulação são fixadas. Naturalmente, são consideradas fibras ópticas com apenas um núcleo. As estratégias de alocação de espectro implementadas, ou seja, as classes do modelo proposto, são: FF, LF, RF, EF, BF, PP, DP e FLEF. Essas estratégias foram escolhidas por serem as mais divulgadas na literatura.

\subsection{Volume de dados}

Os 8 algoritmos mencionados acima foram avaliados em 16 cargas, no intervalo de carga de 50 a 800 Erlangs com passo de 50 Erlangs. Foram utilizadas 5 sementes aleatórias para garantir a variabilidade estatística dos resultados. Dessa forma, o número de estados da rede utilizados para treinamento e teste foram:

$$
\begin{aligned}
\text { ConjuntoDeEntrada } & =\text { algoritmos } * \text { momentos } * \text { cargas } * \text { sementes } \\
& =8 * 90 * 14 * 5 \\
& =50.400
\end{aligned}
$$


O simulador ONS foi adaptado para gerar arquivos de texto contendo as matrizes binárias que representavam os estados da rede. Esses arquivos ocuparam mais de 10 GB de espaço em disco.

\subsection{Redução de Dimensionalidade}

Conforme discutido na Seção 4.2, cada entrada possui 27.520 características (atributos) que vão identificar de qual estratégia de alocação de espectro aquele estado da rede se aproxima mais. Porém, essa alta dimensionalidade costuma implicar não apenas no elevado custo computacional no treinamento do classificador, mas também em prejuízos na sua precisão.

Para reduzir a dimensionalidade, o modelo proposto utiliza a distribuição Quiquadrado para avaliar a relação entre cada atributo e seu valor esperado. Em seguida seleciona-se apenas uma porcentagem dos atributos com os melhores scores de Quiquadrado. São avaliados 20 valores percentuais, variando entre $5 \%$ e $100 \%$, com passo de 5. O parâmetro para escolha da melhor porcentagem foi a acurácia final do modelo, que ocorreu em $40 \%$. Dessa forma, o modelo seleciona as $40 \%$ melhores características, isto é, 11.008 atributos.

\subsection{Hiperparâmetros da Rede Neural}

A primeira camada da rede neural possui, de acordo com a redução de dimensionalidade discutida na Seção anterior, 11.008 neurônios. Após diversos testes (omitidos por questão de espaço) variando a quantidade de neurônios e camadas, o modelo utilizou 5 camadas ocultas com 100,160, 200, 160 e 120 neurônios em cada uma delas, respectivamente. A camada de saída apresenta 8 neurônios, de acordo com as 8 classes (estratégias) os quais podem ser classificados.

A função de ativação para a camada de saída é a Softmax, que retorna a probabilidade de cada classe e, nas demais camadas, a função de ativação utilizada foi a função retificadora linear (Rectified Linear Unit - ReLU) [Goodfellow et al. 2016]. Essas funções de ativação mostraram um melhor resultado comparativamente às outras avaliadas: Tangente Hiperbólica, Sigmoide, ReLU Paramétrica e Swish.

Para reduzir o overfitting utiliza-se a técnica de dropout. Diversos testes foram realizados para os valores $5,10,15,20,25$ e $30 \%$, os melhores resultados encontrados foram com o valor de $20 \%$. Dessa forma, apenas $80 \%$ dos neurônios de cada camada escondida foram considerados em cada iteração na fase de aprendizado da rede neural.

Com relação à taxa de aprendizado, os algoritmos SGD (Stochastic Gradient Descent), RMSprop, Adadelta, Adam, Adamax e Nadam foram testados. O algoritmo de descida de gradiente com taxa de aprendizado adaptativa Adam foi escolhido, tendo em vista que ele apresentou os melhores resultados [Kingma and Ba 2014].

\section{Resultados}

A rede neural descrita na seção anterior foi desenvolvida na linguagem de programação Python com o auxílio da biblioteca Keras [Keras 2019]. As curvas de aprendizado nas fases de treinamento e de validação da rede neural estão mostradas na Figura 4, onde foram utilizados o erro e a acurácia como métricas. A matriz de confusão da RN está mostrada na Tabela 2. 
Tabela 2. Matriz de Confusão.

\begin{tabular}{|c|c|c|c|c|c|c|c|c|c|}
\hline & \multicolumn{8}{|c|}{ Classe Prevista } \\
\hline & & FF & LF & $\mathrm{RF}$ & EF & $\mathrm{BF}$ & PP & DP & FLEF \\
\hline \multirow{8}{*}{$\mid \begin{array}{l}\bar{\sigma} \\
\tilde{U} \\
0 \\
\tilde{D} \\
\tilde{J} \\
\tilde{U}\end{array}$} & FF & 1232 & 0 & 0 & 94 & 0 & 0 & 0 & 0 \\
\hline & LF & 0 & 1242 & 1 & 0 & 2 & 0 & 0 & 0 \\
\hline & $\mathrm{RF}$ & 0 & 0 & 1238 & 12 & 19 & 0 & 0 & 0 \\
\hline & EF & 0 & 0 & 13 & 1172 & 3 & 0 & 0 & 0 \\
\hline & $\mathrm{BF}$ & 0 & 0 & 25 & 1 & 1239 & 0 & 0 & 1 \\
\hline & PP & 0 & 0 & 0 & 0 & 0 & 1245 & 0 & 1 \\
\hline & DP & 0 & 0 & 0 & 2 & 0 & 0 & 1251 & 0 \\
\hline & FLEF & 0 & 1 & 0 & 0 & 17 & 0 & 0 & 1261 \\
\hline
\end{tabular}

A matriz de confusão é uma tabela que mostra as frequências de classificação de cada classe do modelo na fase de teste. Ou seja, a rede neural foi treinada com $80 \%$ das amostras, enquanto que os demais $20 \%$ foram utilizados para testes, e a matriz de confusão evidencia os resultados das predições. Pode-se extrair algumas informações importantes dela, como por exemplo os conceitos de Verdadeiro Positivo (VP), Falso Positivo (FP), Verdadeiro Negativo (VN) e Falso Negativo (FN).

Para um problema de classificação com múltiplas classes, como é o caso deste trabalho, deve-se analisar para cada classe a quantidade de vezes que o classificador acerta (que seriam os VP e os VN) e onde o classificador erra com mais frequência, ou seja, os casos em que a classe real é confundida com outra classe.

$\mathrm{Na}$ matriz de confusão a diagonal principal representa os algoritmos que foram classificados corretamente, ou seja, os Verdadeiros Positivos (VP) e os Verdadeiros Negativos (VN). Por exemplo, a respeito do algoritmo LF pode-se fazer a seguinte análise:

- O classificador obteve êxito ao classificar 1242 amostras como sendo a estratégia de alocação de espectro LF;

- Em 1 amostra o classificador previu erroneamente que o algoritmo era o RF, e em 2 amostras o modelo previu também erroneamente que o estado de rede pertencia ao algoritmo BF. Nesses 3 o classificar errou, tendo em vista que o estado de rede era relativo ao algoritmo LF.

- Por fim, em apenas 1 amostra o classificador previu como LF um estado de rede que pertencia ao algoritmo FLEF.

A análise acima pode ser feita para cada um dos algoritmos. Apesar da baixa quantidade de erros cometidos pelo modelo, o ponto de maior equívoco ocorre entre os algoritmos FF e EF. Isto ocorre devido a difícil tarefa de encontrar faixas de slots disponíveis de tamanho exato, e dessa forma, o algoritmo busca, em muitos os casos, a faixa com maior diferença entre slots livres e solicitados que em grande parte coincide com a estrategia FF.

Três das principais métricas na avaliação de modelos de aprendizado de máquina são a acurácia, a precisão e o recall (revocação). A precisão é mais comumente utilizada em problemas onde os Falsos Positivos são considerados mais prejudiciais que os Falsos Negativos. O recall é mais comumente utilizado em problemas onde Falsos Negativos são considerados mais prejudiciais do que Falsos Positivos. A investigação dessas duas métricas está mais relacionada em problemas de classificação desbalanceados. Já o problema proposto, além de trabalhar com classes balanceadas, não priorizamos FN ou FP. 
Dessa forma, utiliza-se a acurácia como a métrica que indica o comportamento geral do modelo proposto. A acurácia é definida como segue:

$$
\text { Acurácia }=\frac{V P+V N}{V P+V N+F P+F N}=\frac{9.880}{10.072}=98,1 \%
$$

Como pode ser observado na Equação 2, a acurácia na fase de validação foi de $98,1 \%$, um pouco superior à acurácia observada na fase de treinamento: $96,2 \%$. A evolução da acurácia ao longo das épocas de treinamento, bem como o comportamento do erro, podem ser observados na Figura 4.

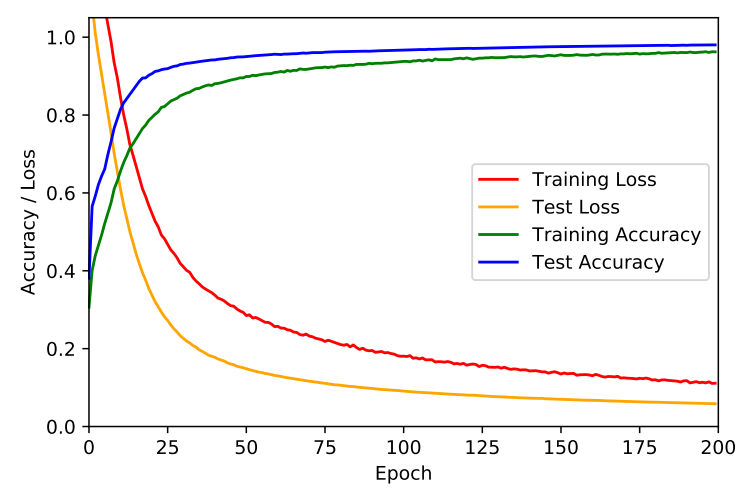

Figura 4. Erro e acurácia nas fases de treinamento e validação da rede neural.

\section{Conclusões e Trabalhos Futuros}

A motivação para este trabalho é a criação de um algoritmo RMLSA que utilize estratégias adaptativas, ou seja, que durante o funcionamento da rede o algoritmo possa ir mudando suas estratégias, de acordo com o tráfego, com intuito de diminuir a probabilidade de bloqueio na rede. Para atingir este objetivo, alguns passos são necessários. O primeiro deles, descrito nesse trabalho, foi verificar se era possível criar um classificador que, a partir de uma amostra do espectro (estado da rede), ser capaz de identificar de qual algoritmo ela pertence. De inicio, foram fixados a estratégia de roteamento como o menor caminho e a escolha da modulação como sendo BPSK. Foram avaliados 8 estratégias de alocação de espectro bem conhecidos na literatura. Para criação do classificador diversas técnicas de aprendizado profundo forma utilizadas. Os dados foram gerados a partir do simulador ONS e totalizaram mais de 10 GB de informação, valor relevante considerandose que os arquivos gerados continham apenas matrizes binárias. O modelo apresentou uma acurácia superior a $98 \%$, mostrando a viabilidade dessa etapa inicial da proposta.

O passo seguinte é verificar o quão genérico é o classificador proposto. Em outras palavras: i) testá-lo com cargas as quais ele não foi treinado, situação na qual esperase que o classificador consiga identificar o algoritmo corretamente; ii) e testá-lo com algoritmos os quais ele também não foi treinado. Nesse segundo caso espera-se poder associar o comportamento do classificador com alguma métrica de eficiência, certamente relacionada à probabilidade de bloqueio. Assim, poderia-se gerar um modelo que estaria apto a informar, durante o funcionamento da rede óptica, se aquelas estratégias adotadas estão garantindo um desempenho eficiente ou não. Por fim, de posse desse classificador de eficiência da rede, propõe-se um algoritmo RMLSA que utilize essa informação provida em tempo real para dinamicamente adaptar as estratégias e garantir um funcionamento mais eficiente (menor taxa de bloqueio) da rede óptica. 


\section{Referências}

Araujo, D. R. B., Bastos-filho, C. J. A., and Martins-filho, J. F. (2015). Methodology to obtain a fast and accurate estimator for blocking probability of optical networks. IEEE/OSA Journal of Optical Communications and Networking, 7(5):380-391.

Barletta, L., Giusti, A., Rottondi, C., and Tornatore, M. (2017). QoT estimation for unestablished lighpaths using machine learning. In 2017 Optical Fiber Communications Conference and Exhibition $(O F C)$, pages 1-3.

Boutaba, R., Salahuddin, M. A., Limam, N., Ayoubi, S., Shahriar, N., Estrada-Solano, F., and Caicedo, O. M. (2018). A comprehensive survey on machine learning for networking: evolution, applications and research opportunities. Journal of Internet Services and Applications, 9(1):16.

Chatterjee, B., Sarma, N., and Oki, E. (2015). Routing and spectrum allocation in elastic optical networks: A tutorial. IEEE Communications Surveys Tutorials, 17(3):1776-1800.

Chatterjee, B. C., Fadini, W., and Oki, E. (2016). A spectrum allocation scheme based on first-lastexact fit policy for elastic optical networks. Journal of Network and Computer Applications, $68: 164-172$.

Chen, X., Guo, J., Zhu, Z., Proietti, R., Castro, A., and Yoo, S. J. B. (2018). Deep-RMSA: A deepreinforcement-learning routing, modulation and spectrum assignment agent for elastic optical networks. In 2018 Optical Fiber Communications Conference and Exposition (OFC), pages $1-3$.

Costa, L. and Drummond, A. (2017). Novo Esquema para Provisão de Modulação Adaptativa em Redes ópticas Elásticas. In SBRC 2017, Belém, Pará.

Costa, L. R. and Drummond, A. C. (2019). ONS - optical network simulator. https: //ons-simulator.com.

Goodfellow, I., Bengio, Y., and Courville, A. (2016). Deep learning. MIT press.

Jia, W., Xu, Z., Ding, Z., and Wang, K. (2016). An efficient routing and spectrum assignment algorithm using prediction for elastic optical networks. In 2016 International Conference on Information System and Artificial Intelligence (ISAI), pages 89-93.

Keras (2019). Keras: The python deep learning library. https://keras.io/. Accessed: 2019-10-04.

Kingma, D. P. and Ba, J. (2014). Adam: A method for stochastic optimization.

Lacerda, J., Fontinele, A., Campelo, D., and Soares, A. (2017). Proteção dedicada para redes Ópticas elásticas considerando efeitos de camada física. In SBRC 2017, Belém, Pará.

Leiva, A., Pavez, N., Beghelli, A., and Olivares, R. (2014). A joint rsa algorithm for dynamic flexible optical networking. In 2014 IEEE Latin-America Conference on Communications (LATINCOM), pages 1-6.

Mata, J., de Miguel, I., Durán, R. J., Merayo, N., Singh, S. K., Jukan, A., and Chamania, M. (2018). Artificial intelligence (AI) methods in optical networks: A comprehensive survey. Optical Switching and Networking, 28:43 - 57. 
Morales, F., Ruiz, M., Gifre, L., Contreras, L. M., Lopez, V., and Velasco, L. (2017). Virtual network topology adaptability based on data analytics for traffic prediction. IEEE/OSA Journal of Optical Communications and Networking, 9(1):A35-A45.

Muhammad, A., Zervas, G., and Forchheimer, R. (2015). Resource allocation for space-division multiplexing: Optical white box versus optical black box networking. Journal of Lightwave Technology, 33(23):4928-4941.

Musumeci, F., Rottondi, C., Nag, A., Macaluso, I., Zibar, D., Ruffini, M., and Tornatore, M. (2018). An overview on application of machine learning techniques in optical networks. IEEE Communications Surveys \& Tutorials, 21(2):1383-1408.

Proietti, R., Chen, X., Castro, A., Liu, G., Lu, H., Zhang, K., Guo, J., Zhu, Z., Velasco, L., and Yoo, S. J. B. (2018). Experimental demonstration of cognitive provisioning and alien wavelength monitoring in multi-domain EON. In 2018 Optical Fiber Communications Conference and Exposition (OFC), pages 1-3.

Rosa, A., Cavdar, C., Carvalho, S., Costa, J., and Wosinska, L. (2012). Spectrum allocation policy modeling for elastic optical networks. In High Capacity Optical Networks and Emerging/Enabling Technologies, pages 242-246.

Tessinari, R. S., Colle, D., and Garcia, A. S. (2018). Cognitive zone-based spectrum assignment algorithm for elastic optical networks. In 2018 International Conference on Optical Network Design and Modeling (ONDM), pages 112-117.

Tode, H. and Hirota, Y. (2016). Routing, Spectrum and Core Assignment on SDM Optical Networks (Invited). 9(1):Tu2H.1.

Vela, A. P., Ruiz, M., Fresi, F., Sambo, N., Cugini, F., Meloni, G., Potì, L., Velasco, L., and Castoldi, P. (2017). Ber degradation detection and failure identification in elastic optical networks. Journal of Lightwave Technology, 35(21):4595-4604.

Velasco, L., Vela, A. P., Morales, F., and Ruiz, M. (2017). Designing, operating, and reoptimizing elastic optical networks. Journal of Lightwave Technology, 35(3):513-526.

Waldman, H. (2018). The impending optical network capacity crunch. In 2018 SBFoton International Optics and Photonics Conference (SBFoton IOPC), pages 1-4.

Walkowiak, K., Klinkowski, M., and Lechowicz, P. (2018). Dynamic routing in spectrally spatially flexible optical networks with back-to-back regeneration. IEEE/OSA Journal of Optical Communications and Networking, 10(5):523-534.

Wang, R. and Mukherjee, B. (2012). Spectrum management in heterogeneous bandwidth networks. In 2012 IEEE Global Communications Conference (GLOBECOM), pages 29072911.

Yan, S., N Khan, K., Mavromatis, A., Gkounis, D., Fan, Q., Ntavou, F., Nikolovgenis, K., Guo, C., Meng, F., Hugues-Salas, E., Lu, C., Pak Tau Lau, A., Nejabati, R., and Simeonidou, D. (2017). Field trial of machine-learning-assisted and sdn-based optical network planning with network-scale monitoring database. page TH.PDP.B4. 